\section{Hartree-Fock Calculations of the Interaction Potential Parameters for Noble-Gas Atoms}

4. Bielski and J. Wasilewski

Institute of Physics, Nicholas Copernicus University, Toruń, Poland

Z. Naturforsch. 35a, 1112-1115 (1980);

received December 24,1979

The interaction potential parameters $\left(\left\langle r^{k}\right\rangle\right.$, Hindmarsh's radius $r_{\mathrm{H}}, C_{6}, C_{8}, C_{12}$ ) are calculated for the ground and some excited configurations of the noble-gas atoms from the Hartree-Fock functions. A formula for the $C_{8}$ constant is derived.

\section{Introduction}

The phenomenon of the pressure broadening of spectral lines is actually the main source of information about interactions between the groundand excited-state atoms in the gas phase. In the last few years the quantum theory of the pressure line broadening has been widely developed. However, in order to compare the results of these theories with experiment the detailed knowledge of the interaction potential between the emitter (excited-state atom) and the perturber (groundstate atom) is necessary. These potentials are precisely known in only very few cases. Therefore different approximate formulae, such as the model function of the Lennard-Jones type, are rather frequently applied. These model potentials were shown to be very important in many applications of the line broadening studies, first of all in astrophysics and plasma diagnostics (cf. e.g. [4]).

In this paper we present the results of HartreeFock calculations of some basic atomic parameters, appearing in these approximate formulae. These parameters can be used to estimate the interaction energies of the ground-state noble-gas atoms with other atoms. In the case of neon atom we present also the corresponding parameters for excited configurations, because the pressure broadening of the neon spectral lines is studied experimentally in our laboratory. In this case an estimate of the $C_{6}$, $C_{8}$ and $C_{12}$ constants for the interaction of excited neon with the ground-state neon and helium atoms

Reprint request to Dr. A. Bielski, Institute of Physics, Nicholas Copernicus University, Grudziadzka 5, 87-100 Torun, Polen. was also possible. Despite recent progress in the interaction-constant calculations (cf. e.g. [8]) it seems, that some simple methods of their estimation, particulary for the excited states, are still of value for experimentalists. Our own derivation of the $C_{8}$ interaction constant is also presented.

\section{Interaction Energy}

The first two terms in the hamiltonian of two atoms interacting at long distance, in the moleculeoriented coordinate system ( $z$-axis along the line emitter-perturber) have the form [1]:

$$
\begin{aligned}
V\left(R_{\mathrm{p}}, \boldsymbol{r}\right) \equiv & H^{\prime}+H^{\prime \prime} \\
= & \left(e^{2} / R_{\mathrm{p}}^{3}\right)\left(x x_{\mathrm{p}}+y y_{\mathrm{p}}-2 z z_{\mathrm{p}}\right) \\
& +\frac{3}{2}\left(e^{2} / R_{\mathrm{p}}^{4}\right) \\
& \cdot\left(r^{2} z_{\mathrm{p}}-z r_{\mathrm{p}}^{2}+2 x z x_{\mathrm{p}}\right. \\
& +2 y z y_{\mathrm{p}}-3 z^{2} z_{\mathrm{p}} \\
& -2 x x_{\mathrm{p}} z_{\mathrm{p}}-2 y y_{\mathrm{p}} z_{\mathrm{p}} \\
& \left.+3 z z_{\mathrm{p}}^{2}\right) .
\end{aligned}
$$

Here, $R_{\mathrm{p}}$ is the interatomic (emitter-perturber) distance, the unsubscripted coordinates measure the electronic position relative to the emitter nucleus, the p-subscripted ones its position relative to the perturber nucleus.

Within the second-order perturbation theory the first of these two terms gives us the well-known Van-der-Waals potential, $-C_{6} R_{\mathrm{p}}^{-6}$. Assuming the perturber atom, e.g. the noble-gas one, in the ${ }^{1} S_{0}$ ground state, and the $|K J M\rangle$ state of the emitter ( $K$ represents the set of other quantum numbers necessary), the $C_{6}$ interaction constant can be expressed as, ef. $[2,3]$ :

$$
\begin{gathered}
C_{6}(K J M)=\frac{\sigma_{\mathrm{p}} I_{\mathrm{p}}}{2(2 J+1)} \\
\cdot \sum_{K^{\prime} J^{\prime}} \frac{S\left(K J, K^{\prime} J^{\prime}\right)\left[1+3\left(1 J^{\prime} 0 M \mid J M\right)^{2}\right]}{E_{K^{\prime} J^{\prime}}-E_{K J}+I_{\mathrm{p}}} .
\end{gathered}
$$

In this formula the summation runs over all the states $\left|K^{\prime} J^{\prime}\right\rangle$ of the emitter, $E_{K J}, E_{K^{\prime} J^{\prime}}$ are the corresponding state energies and $S\left(K J, K^{\prime} J^{\prime}\right)$ the dipole line strengths. Other quantities concern the perturber atom: $\sigma_{\mathrm{p}}$ is its dipole poalizability, $I_{\mathrm{p}}$ the ionization potential; $\left(1 J^{\prime} 0 M \mid J M\right)$ is the Clebsh-Gordan coefficient. 
On the same level of approximation, assuming still the ${ }^{1} S_{0}$ state for the perturber, one obtains from the second part of (1) the $-C_{8} R_{\mathrm{p}}^{-8}$ term of the long-range potential, with the force constant given by

$$
\begin{aligned}
C_{8}\left(K J M ; K_{\mathrm{p}} J_{\mathrm{p}}\right)= & \frac{9}{4} e^{2} \sum_{K^{\prime} J^{\prime} M^{\prime}} \sum_{K_{\mathrm{p}}^{\prime} J_{\mathrm{p}}^{\prime}} \frac{1}{E_{K^{\prime} J^{\prime}}-E_{K J}+E_{K_{\mathrm{p}}^{\prime} J_{\mathrm{p}}^{\prime}}-E_{K_{\mathrm{p}} J_{\mathrm{p}}}} \\
& +\left\{\frac { 1 } { 3 } S _ { ( \mathrm { d } ) } ( K _ { \mathrm { p } } J _ { \mathrm { p } } , K _ { \mathrm { p } } ^ { \prime } J _ { \mathrm { p } } ^ { \prime } ) \left[\left|\left\langle K J M\left|r^{2}\right| K^{\prime} J^{\prime} M^{\prime}\right\rangle\right|^{2}+9\left|\left\langle K J M\left|z^{2}\right| K^{\prime} J^{\prime} M^{\prime}\right\rangle\right|^{2}\right.\right. \\
& \left.+4\left|\left\langle K J M|x z| K^{\prime} J^{\prime} M^{\prime}\right\rangle\right|^{2}+4\left|\left\langle K J M|y z| K^{\prime} J^{\prime} M^{\prime}\right\rangle\right|^{2}\right] \\
& +\frac{2}{5} S_{(\mathrm{q})}\left(K_{\mathrm{p}} J_{\mathrm{p}}, K_{\mathrm{p}}^{\prime} J_{\mathrm{p}}^{\prime}\right)\left[\left|\left\langle K J M|x| K^{\prime} J^{\prime} M^{\prime}\right\rangle\right|^{2}\right. \\
& \left.\left.+\left|\left\langle K J M|y| K^{\prime} J^{\prime} M^{\prime}\right\rangle\right|^{2}+3\left|\left\langle K J M|z| K^{\prime} J^{\prime} M^{\prime}\right\rangle\right|^{2}\right]\right\} .
\end{aligned}
$$

Here, the second sum concerns the $\left|K_{\mathrm{p}}^{\prime} J_{\mathrm{p}}^{\prime}\right\rangle$ states of the perturber, $E_{K_{\mathrm{p}} J_{\mathrm{p}}}, E_{K_{\mathrm{p}} J_{\mathrm{p}}^{\prime}}$ are the corresponding perturber state energies and $S_{(\mathrm{d})}, S_{(\mathrm{q})}$ are the line strengths for, respectively, the dipole and quadrupole transitions in the perturber atom. After application of the Wigner-Eckart theorem, taking into consideration the definition of polarizabilities and assuming (as in the case of the $C_{6}$ expression) that the energy differences $E_{K_{\mathrm{p}}{ }^{\prime} J_{\mathrm{p}}{ }^{\prime}}-E_{K_{\mathrm{p}} J_{\mathrm{p}}}$ are approximately equal to the ionization potential $I_{\mathrm{p}}$ of the perturber, we obtain the following approximate formula:

$$
\begin{aligned}
& C_{8}(K J M)= \frac{9}{4} \frac{I_{\mathrm{p}}}{(2 J+1)} \sum_{K^{\prime} J^{\prime}} \frac{1}{E_{K^{\prime} J^{\prime}}-E_{K J}+I_{\mathrm{p}}} \\
& \cdot\left\{\sigma _ { \mathrm { p } } S _ { ( \mathrm { q } ) } ( K J , K ^ { \prime } J ^ { \prime } ) \left[3\left(2 J^{\prime} O M \mid J M\right)^{2}\right.\right. \\
&\left.+\left(2 J^{\prime}-1 M+1 \mid J M\right)^{2}+\left(2 J^{\prime} 1 M-1 \mid J M\right)^{2}\right] \\
&\left.+\frac{3}{5} \sigma_{\mathrm{p}(\mathrm{q})} S\left(K J, K^{\prime} J^{\prime}\right)\left[1+2\left(1 J^{\prime} O M \mid J M\right)^{2}\right]\right\} .
\end{aligned}
$$

where $S_{(\mathrm{q})}\left(K J, K^{\prime} J^{\prime}\right)$ is the line strengths for the quadrupole transitions in the emitter, $\sigma_{\mathrm{p}(\mathrm{q})}$ is the quadrupole polarizability of the perturber, all the other quantities have been discussed previously.

It is rather difficult to use the above written formulae $(2,4)$ for calculations of the $C_{6}$ and $C_{8}$ interaction constants, because one should know the line strengths $S\left(K J, K^{\prime} J^{\prime}\right), S_{(q)}\left(K J, K^{\prime} J^{\prime}\right)$ for all the lines corresponding to the transitions from the $|K J\rangle$ state of the emitter. For this reason some more approximate formulae are in common use. The $C_{6}$ constant can be calculated in the Unsöld approximation [4] as

$$
C_{6}=e^{2} \sigma_{\mathrm{p}}\left\langle r^{2}\right\rangle \text {. }
$$

For the $C_{8}$ constant the approximate formula of Davison [5] is known:

$$
C_{8}=\frac{3}{2} e^{2} \sigma_{\mathrm{p}}\left\langle r^{4}\right\rangle+\frac{5}{2} e^{2} \sigma_{\mathrm{p}(\mathrm{q})}\left\langle r^{2}\right\rangle .
$$

In these expressions $\left\langle r^{k}\right\rangle$ means the average value of the corresponding power of the optical electron radial coordinate (relative to the emitter nucleus).

The Van-der-Waals interaction energy,

$$
\Delta E\left(R_{\mathrm{p}}\right)=-C_{6} R_{\mathrm{p}}^{-6}-C_{8} R_{\mathrm{p}}^{-8}
$$

includes only the attractive forces. In many cases the pressure line broadening can be correctly described using only the first term of (7). However in some cases (e.g. when the helium atom is used as the perturber) the inclusion of repulsive forces becomes necessary. The simplest way to do this is the application of the Lennard-Jones potential

$$
\begin{aligned}
& \Delta E\left(R_{\mathrm{p}}\right)=C_{12} R_{\mathrm{p}}^{-12}-C_{6} R_{\mathrm{p}}^{-6}, \quad \text { or } \\
& \Delta E\left(R_{\mathrm{p}}\right)=C_{12} R_{\mathrm{p}}^{-12}-C_{6} R_{\mathrm{p}}^{-6}-C_{8} R_{\mathrm{p}}^{-8} .
\end{aligned}
$$

Hindmarsh et al. [6] gave an empirical method of estimation of the $C_{12}$ constant. According to these authors

$$
C_{12}=q\left(r_{\mathrm{H}}+r_{\mathrm{H}_{\mathrm{p}}}\right)^{12},
$$

where $q$ is an empirical constant and $r_{\mathrm{H}}, r_{\mathrm{H}_{\mathrm{p}}}$ are the "Hindmarsh's radii" of emitter and perturber, respectively. These Hindmarsh's radii can be easily extracted from the long-distance shape of the radial electron density, as described in [6].

\section{Numerical Calculations and Discussion}

For the calculations of the expectation values $\left\langle r^{2}\right\rangle,\left\langle r^{4}\right\rangle(5,6)$ and the Hindmarsh's radii $r_{\mathrm{H}}(9)$ we have used the numerical Hartree-Fock orbitals. The Hartree-Fock equations for the ground states of the noble-gas atoms, $\mathrm{He}$ to $\mathrm{Xe}$, and for some excited configurations of the neon atom have been 
solved using the numerical MCHF program of Froese Fischer, cf. [7]. For excited configurations all the orbitals were made selfconsistent, the frozencore approach has not been used. The average energy expression of the one-configurational approach has been considered only; it corresponds to the ${ }^{1} S_{0}$ term energy for the closed-shell ground states, for the open-shell configurations it represents the properly weighted sum of the SL-term energies.

The values of $\left\langle r^{k}\right\rangle$ for every $n l$-orbital can be obtained directly from the MCHF program. The values of $r_{\mathrm{H}}$ (9) have been calculated by Hindmarsh's approach, using the radial electron density constructed from numerical orbitals. Despite of the fact that the radial one-electron density depends on all the atomic orbitals, the analysis we have performed indicates that the quantities $\left\langle r^{k}\right\rangle$ and $r_{\mathrm{H}}$ for the expressions $(5,6,9)$ should be calculated for the optical electron atomic orbital only, in accordance with the common practice, cf. e.g. [9] and the discussion in [8]. In the case of Hindmarsh's radius the inner shells contribute only about $10^{-7}$ of the value, calculated for the valence orbital separately. In the case of $\left\langle r^{k}\right\rangle$ the correct expectation value, in accordance with the average energy expression used, is

$$
\left\langle r^{k}\right\rangle=\sum_{(n l)} \frac{N_{(n l)}}{N}\left\langle r^{k}\right\rangle_{(n l)},
$$

where $\left\langle r^{k}\right\rangle_{(n l)}$ denotes the $n l$-orbital expectation value, $N_{(n l)}$ is the population of the $n l$-shell, $N$ is the total number of electrons, $N=\sum_{(n l)} N_{(n l)}$. However, the application of $\left\langle r^{k}\right\rangle$ values given by (10) in the formulae $(5,6)$ results in complete disagreement between the calculated and experimental coefficients of pressure broadening for the neon lines investigated. This means that the Unsöld approximation [4], formulated originally for the hydrogen atom, should be treated as concerning only the single valence electron, even when transferred to the many-electron atoms.

The results of our calculations are presented in Tables I and II. Table I contains the values of $\left\langle r^{2}\right\rangle,\left\langle r^{4}\right\rangle$ and $r_{\mathrm{H}}$ for the ground-state noble-gas atoms, helium to xenon. In Table II the same quantities are presented for different excited configurations of neon. Here, we have calculated also the $C_{6}, C_{8}$ and $C_{12}$ constants according to (5), (6) and (9), respectively, for the interactions between the excited neon atom and non-excited neon and helium atoms. We have used the values from $[10]$ as the static dipole polarizabilities $\sigma_{\mathrm{p}}$, and the values calculated by Dalgarno [11] as the static quadrupole polarizabilities $\sigma_{\mathrm{p}(\mathrm{q})}$. For both the

Table 1. Expectation values of the $r^{2}$ and $r^{4}$ operators for the outermost $n p$-orbital, and the Hindmarsh's radii $r_{\mathbf{H}}$, for the ground-state noble-gas atoms (atomic units).

\begin{tabular}{|c|c|c|c|c|}
\hline & \multirow[t]{2}{*}{$\left\langle r^{2}\right\rangle^{\mathrm{a}}$} & \multirow[t]{2}{*}{$\left\langle r^{4}\right\rangle$} & \multicolumn{2}{|l|}{$r_{\mathrm{H}}$} \\
\hline & & & $\begin{array}{l}\text { this } \\
\text { paper }\end{array}$ & $\begin{array}{l}\text { Hindmarsh } \\
\text { et al. [6] }\end{array}$ \\
\hline $\mathrm{He}$ & 1.184628 & 3.887947 & 3.3599 & 3.31 \\
\hline $\mathrm{Ne}$ & 1.228456 & 3.908740 & 3.8415 & 3.97 \\
\hline $\mathrm{Ar}$ & 3.310850 & 20.91084 & 5.1902 & 5.29 \\
\hline $\mathrm{Kr}$ & 4.455077 & 35.22926 & 5.6960 & 5.86 \\
\hline $\mathrm{Xe}$ & 6.276875 & 65.22770 & 6.3989 & - \\
\hline
\end{tabular}

a cf. also [7], Table $2-3$.

Table 2. Expectation values of the $r^{2}$ and $r^{4}$ operators for the singly occupied valence orbital, and the Hindmarsh's radii $r_{\mathrm{H}}$, for excited configurations of neon, together with the interaction potential constants $C_{6}, C_{8}, C_{12}$ for the interactions $\mathrm{Ne}^{*}-\mathrm{Ne}$ and $\mathrm{Ne}^{*}-\mathrm{He}$.

\begin{tabular}{|c|c|c|c|c|c|c|c|c|c|}
\hline \multirow{2}{*}{$\begin{array}{l}\text { Configu- } \\
\text { ration }\end{array}$} & \multirow[b]{2}{*}{$\left\langle r^{2}\right\rangle^{a}$} & \multirow[b]{2}{*}{$\left\langle r^{4}\right\rangle^{\mathrm{a}}$} & \multirow[b]{2}{*}{$r_{\mathrm{H}^{2}}^{\mathrm{a}}$} & \multicolumn{3}{|c|}{$\mathrm{Ne}^{*}-\mathrm{Ne}$} & \multicolumn{3}{|c|}{$\mathrm{Ne}^{*}-\mathrm{He}$} \\
\hline & & & & $C_{6} \mathrm{~b}$ & $C_{8} \mathrm{c}$ & $C_{12}^{\mathrm{d}}$ & $C_{6}{ }^{\mathrm{b}}$ & $C_{8}{ }^{\mathrm{c}}$ & $C_{12}^{\mathrm{d}}$ \\
\hline$\ldots 2 \mathrm{p}^{5} 3 \mathrm{~s}$ & 22.908 & 902.9526 & 9.3449 & 0.582 & 0.011 & $1.19_{10}-102$ & 0.319 & 0.00564 & $7.64_{10}-103$ \\
\hline $4 \mathrm{~s}$ & 140.25 & 28714.00 & 19.292 & 3.567 & 0.315 & $1.02_{10}-99$ & 1.956 & 0.17 & $7.89_{10}-100$ \\
\hline $5 \mathrm{~s}$ & 485.99 & 323555.4 & 35.550 & 12.36 & 3.48 & $2.33_{10}-97$ & 6.776 & 1.89 & $1.99_{10}-97$ \\
\hline $6 \mathrm{~s}$ & 1253.5 & 2088266. & 48.943 & 31.88 & 22.4 & $2.02_{10}-95$ & 17.48 & 12.2 & $1.81_{10}-95$ \\
\hline $7 \mathrm{~s}$ & 2696.2 & 9505023. & 68.761 & 68.57 & 101.6 & $9.27_{10}-94$ & 37.59 & 56.6 & $8.56_{10}-94$ \\
\hline$\ldots 2 p^{5} 3 p$ & 44.499 & 3370.27 & 12.378 & 1.132 & 0.0386 & $1.43_{10}-101$ & 0.620 & 0.0204 & $9.98_{10}-102$ \\
\hline $4 p$ & 232.68 & 78354.4 & 24.318 & 5.918 & 0.85 & $1.07_{10}-98$ & 3.244 & 0.462 & $8.74_{10}-99$ \\
\hline $5 \mathrm{p}$ & 726.91 & 721377.6 & 38.824 & 18.49 & 7.74 & $1.57_{10}-96$ & 10.14 & 4.23 & $1.37_{10}-96$ \\
\hline$\ldots 2 \mathrm{p}^{5} 3 \mathrm{~d}$ & 52.452 & 6443.05 & 14.504 & 1.334 & 0.0719 & $6.28_{10}-101$ & 0.731 & 0.0385 & $5.46_{10}-101$ \\
\hline $4 d$ & 282.31 & 130487.3 & 27.109 & 7.180 & 1.41 & $3.34_{10}-98$ & 3.936 & 0.768 & $2.77_{10}-98$ \\
\hline $5 \mathrm{~d}$ & 1342.1 & 2453512 . & 51.005 & 34.13 & 26.3 & $3.20_{10}-95$ & 18.71 & 14.4 & $2.88_{10}-95$ \\
\hline
\end{tabular}

Units: a atomic units; b ${ }_{10}-58 \mathrm{erg} \mathrm{\textrm {cm } ^ { 6 }} ;{ }_{10}{ }_{10}-71 \mathrm{erg} \mathrm{\textrm {cm } ^ { 8 }} ; \mathrm{d}$ erg $\mathrm{cm}^{12}$. 
$\mathrm{Ne}{ }^{*}-\mathrm{Ne}$ and $\mathrm{Ne}{ }^{*}-\mathrm{He}$ interactions the potential parameters presented here have recently been used (Refs. [12-15]) for calculations of the pressure broadening coefficients for the neon spectral lines arising from the transitions between the levels belonging to configurations: $2 \mathrm{p}^{5} 3 \mathrm{~s}-2 \mathrm{p}^{5} 3 \mathrm{p}$; $2 \mathrm{p}^{5} 3 \mathrm{p}-2 \mathrm{p}^{5} 4 \mathrm{~d} ; 2 \mathrm{p}^{5} 3 \mathrm{p}-2 \mathrm{p}^{5} 5 \mathrm{~s}$, studied experimentally in our laboratory. A reasonable agreement between the calculated and experimental coefficients has been noted. This indicates that within the approximation level represented by the formulae $(5,6,9)$ the electron correlation effects are of lower importance and the Hartree-Fock approxi-

[1] H. Margenau, Phys. Rev. 40, 382 (1932), Rev. Mod. Phys. 11, 1 (1939).

[2] J. Fiutak and M. Frąckowiak, Bull. Acad. Polon. Sci. Ser. Sci. Math. Astr. Phys. 11, 175 (1963).

[3] A. Bielski and J. Szudy, Z. Naturforsch. 30a, 1903 (1975).

[4] A. Unsold, Physik der Sternatmosphären, Springer Verlag, Berlin 1955.

[5] W. D. Davison, J. Phys. B, 1, 139 (1968).

[6] W. R. Hindmarsh, A. D. Petford, and B. Smith, Proc. Roy. Soc. London A 297, 296 (1967).

[7] C. Froese Fischer, The Hartree-Fock Method for Atoms: A Numerical Approach, Wiley-Intersci. New York 1977.

[8] F. Maeder and W. Kutzelnigg, Chem. Phys. 42, 95 (1979). mation alone can be effectively used for interpretation of the pressure line broadening experiments.

\section{Acknowledgement}

We are grateful to Prof. Dr. W. Kutzelnigg (Bochum) and to Dr. J. Szudy from our Institute for discussions and reading of the manuscript. All the calculations presented here were done on the R-32 computer of the University Computing Center. This work has been partially supported by the Polish Academy of Sciences, Institute for Low Temperature and Structure Research, project No. MR.I.9.

[9] W. R. Hindmarsh and J. M. Farr, Collision Broadening of Spectral Lines by Neutral Atoms, in Progress in Quantum Electronics, Vol. 2, Part 3, edited by J. M. Sanders and S. Stenholm, Pergamon Press, London 1972.

[10] Landolt-Börnstein, Atom und Molekularphysik, Vol.1, 1950 , p. 401.

[11] A. Dalgarno, Adv. Phys. 11, 281 (1962).

[12] A. Bielski and J. Wolnikowski, Acta Phys. Polon. A 54, 601 (1978).

[13] A. Bielski, E. Lisicki, M. Nesterov, and J. Wolnikowski, Acta Phys. Polon A s4, 611 (1978).

[14] A. Bielski, S. Birza, K. Bystra, and W. Dokurno, Physika 97 C, 249 (1979).

[15] A. Bielski, W. Dokurno, J. Szudy, and J. Wolnikowski, Physica 101 C, 113 (1980). 